\title{
Prevalencia de desnutrición en niños al ingreso hospitalario en 9 países latinoamericanos y análisis de sus factores asociados
}

\section{Prevalence of malnutrition in children at hospital admission in 9 Latin American countries and analysis of associated factors}

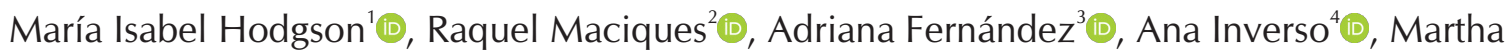

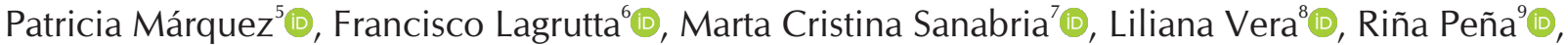
Lázaro Alfonso $\operatorname{Novo}^{10}{ }^{\circ}$, Rubens Feferbaum ${ }^{11}$ [D, Camila Kluever ${ }^{12}$ (B)

${ }^{1}$ Pontificia Universidad Católica de Chile. Facultad de Medicina. Departamento Gastroenterología y Nutrición Pediátrica Santiago, Chile.

${ }^{2}$ Cardiocentro Pediátrico Universitario “William Soler". La Habana, Cuba.

${ }^{3}$ Hospital de Niños Sor Maria Ludovica, La Plata, Argentina.

${ }^{4}$ Hospital Pereira Rosell. Montevideo, Uruguay.

${ }^{5}$ Instituto Nacional de Pediatría. Ciudad de México, México.

${ }^{6}$ Hospital del Niño Doctor José Renán Esquivel. Panamá, Panamá.

${ }^{7}$ Universidad Nacional de Asunción, Facultad de Ciencias Médicas, Hospital de Clínicas. San Lorenzo, Paraguay.

${ }^{8}$ Hospital de Niños José Manuel de los Rios. Caracas, Venezuela.

${ }^{9}$ Hospital General Dr. Juan José Arévalo Bermejo. Guatemala, Guatemala.

${ }^{10}$ Hospital Pediátrico Universitario “William Soler". La Habana, Cuba.

${ }^{11}$ Universidad de São Paulo, Facultad de Medicina, Instituto del Niño. São Paulo, Brasil.

${ }^{12}$ Pontificia Universidad Católica de Chile. Santiago, Chile.

\section{RESUMEN}

Introducción: La desnutrición continúa siendo una causa frecuente de aumento de la morbimortalidad, especialmente en pacientes hospitalizados. Objetivo: Evaluar la tasa de desnutrición infantil observada al ingreso hospitalario en nueve países latinoamericanos y estimar el impacto de diversas condicionantes que pueden incidir en su desarrollo. Materiales y Métodos: Estudio descriptivo, transversal de niños de ambos sexos, de 0 a 18 años de edad, hospitalizados en nueve países latinoamericanos (Argentina, Chile, Cuba, Guatemala, México, Panamá, Paraguay, Uruguay y Venezuela). La información se obtuvo mediante encuesta y antropometría efectuada

\section{ABSTRACT}

Introduction: Malnutrition continues to be a frequent cause of increased morbidity and mortality, especially in hospitalized patients. Objective: To evaluate the observed infant malnutrition rate at hospital admission in nine Latin American countries and to estimate the impact of various conditions that may affect their development. Materials and Methods: This was a descriptive, cross-sectional study of children of both sexes, from 0 to 18 years of age, hospitalized in nine Latin American countries (Argentina, Chile, Cuba, Guatemala, Mexico, Panama, Paraguay, Uruguay and Venezuela). The information was obtained through a survey and anthropometry carried out during

Correspondencia: María Isabel Hodgson, correo: hodgson@med.puc.cl Conflicto de interés: Los autores declaran no tener conflictos de interés Fuente de financiamiento: No hay financiamiento involucrado. Recibido: 29/07/2021 Aceptado: 04/11/2021 DOI: https://doi.org/10.31698/ped.48032021005

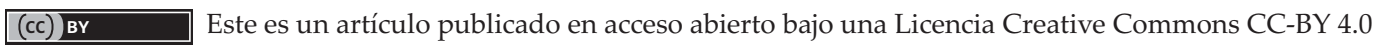


durante las primeras 48 horas de ingresados. Para la evaluación nutricional se evaluó el peso y la talla de los niños de acuerdo a los estándares de la Organización Mundial de la Salud (OMS). El análisis estadístico se realizó en el programa SPSS versión 24. Resultados: Del total de 5.366 pacientes, $45,2 \%$ eran mujeres y $46,5 \%$ menores de 2 años. Un $50,2 \%$ eran eutróficos, un $15,4 \%$ presentaba riesgo de desnutrir o desnutrición leve, un $12,5 \%$ desnutrición, un $14,2 \%$ sobrepeso o riesgo de obesidad, y un $7,7 \%$ obesidad. El 20,5\% eran de talla baja. Los niños con 4 o más hospitalizaciones previas tuvieron mayor riesgo de presentar malnutrición por déficit $(\mathrm{OR}=1,7)$. Las patologías cardiológicas presentaron un $\mathrm{OR}=1,8$ de presentar malnutrición por déficit. Conclusiones: La prevalencia de desnutrición en niños al ingreso hospitalario es moderada y se relaciona especialmente a patología cardiológica y a los antecedentes de varias hospitalizaciones previas.

Palabras claves: Desnutrición, niños hospitalizados, Latinoamérica.

\section{INTRODUCCIÓN}

Las llamadas enfermedades crónicas no transmisibles, se han convertido en la principal causa de muerte y discapacidad en la mayoría de los países de América Latina y el Caribe, relacionados a la obesidad y el sobrepeso. Sin embargo, ladesnutrición continúa siendo una causa frecuente de aumento de la morbimortalidad, especialmente en los pacientes hospitalizados. Más de una tercera parte de las muertes infantiles en todo el mundo se atribuye a la desnutrición, y es uno de los factores que más contribuyen a la carga mundial de morbilidad ${ }^{(1)}$. La pobreza es una de las principales causas de desnutrición primaria y afecta más a las zonas rurales que a las zonas urbanas ${ }^{(2)}$. Un niño que vive en una zona rural tiene entre 1,5 a 3,7 más riesgo de sufrir desnutrición que un niño que vive en zona urbana y 4 veces más probabilidades de sufrirla si es de origen indígena. Los países andinos y centroamericanos son claros ejemplos de esta situación ${ }^{(3)}$.

Otro factor determinante en la desnutrición de los pacientes es la educación. Un buen nivel de educación mejora los conocimientos sobre nutrición y el estado de salud en general. Además, las personas mejor educadas cuidan más de su estado de salud y su nutrición ${ }^{(2)}$. Por lo mismo, un mayor nivel de the first 48 hours after admission. For the nutritional evaluation, the weight and height of the children were evaluated according to the standards of the World Health Organization (WHO). Statistical analysis was performed using SPSS version 24. Results: Of the total of 5,366 patients, $45.2 \%$ were female and $46.5 \%$ were under 2 years of age. $50.2 \%$ were eutrophic, $15.4 \%$ had a risk of malnutrition or mild malnutrition, $12.5 \%$ malnutrition, $14.2 \%$ overweight or at risk of obesity, and $7.7 \%$ were obese. $20.5 \%$ were short. Children with 4 or more previous hospitalizations had a higher risk of presenting deficiency malnutrition $(\mathrm{OR}=1.7)$. Patients with cardiological pathologies presented an $\mathrm{OR}=1.8$ of presenting deficiency malnutrition. Conclusions: The prevalence of malnutrition in children at hospital admission is moderate and is especially related to cardiac pathology and a history of several previous hospitalizations.

Keywords: Malnutrition, hospitalized children, Latin America.

educación en las madres incide directamente en un mejor estado de salud y nutrición de los niños ${ }^{(2)}$.

El efecto del bajo peso de nacimiento se asocia a una mayor prevalencia de desnutrición a corto y mediano plazo (menor peso, talla, circunferencia de cráneo). A largo plazo, todo esto trae consecuencias negativas en el ámbito cognitivo. Más adelante, en la etapa adulta, el bajo peso de nacimiento se asociaría a una mayor prevalencia de enfermedades crónicas no transmisibles (enfermedades cardiovasculares, obesidad y diabetes $)^{(4)}$.

En los niños, otro factor que favorece el desarrollo de desnutrición son las enfermedades. La población pediátrica tiene altos requerimientos de macro y micronutrientes, dado el acelerado crecimiento y desarrollo. Además, tienen bajos depósitos energéticos, y muchas veces presentan inmadurez fisiológica de algunos órganos y falta de autonomía para alimentarse. La enfermedad predispone a la desnutrición por una ingesta deficiente, por alteración de la absorción de nutrientes, el incremento de los requerimientos y el aumento de sus pérdidas. Una de las consecuencias más importantes de la desnutrición es la depresión del sistema inmune ${ }^{(5,6)}$. 
Las cifras de desnutrición en niños hospitalizados varían entre un $20 \%$ a $25 \%$, ya sea por causas quirúrgicas y médicas, porcentajes que aumentan a medida que se prolonga la hospitalización ${ }^{(7-10)}$.

Un estudio en Guadalajara, México, mostró que un $17 \%$ de los niños encuestados en un hospital, presentaban desnutrición aguda y $8 \%$ desnutrición crónica $^{(11)}$. En un estudio realizado en Munich, Alemania en 2008, se observó que $24,1 \%$ de los niños presentaban desnutrición en el momento del ingreso. La mayor prevalencia se observó en los niños de 2 a 5 años de edad ${ }^{(9,12)}$. En Brasil en el 2009. en un estudio efectuado en 10 hospitales pediátricos universitarios, se observó que en el momento del ingreso el $16 \%$ de los niños presentaban una desnutrición moderada-severa y el 30\% tenían una talla baja para su edad, acorde la Organización Mundial de la Salud (OMS) $)^{(8,13)}$. En el mismo año 2009, en la ciudad de Córdoba en Argentina, se registró una prevalencia de desnutrición al ingreso hospitalario del $50,2 \%{ }^{(14)}$.

Dado lo anterior, la prevalencia de desnutrición en la población pediátrica hospitalizada se considera en general elevada, aunque es difícil comparar las distintas series ya que los criterios para diagnosticarla varían entre países. Independiente de lo anterior, las tasas de desnutrición en pacientes hospitalizados suelen ser mayores en países subdesarrollados ${ }^{(6)}$.

Identificar la desnutrición hospitalaria y manejarla adecuadamente, es de importancia para evitar complicaciones, disminuir el tiempo de estancia hospitalaria o la mortalidad en los niños. Existen pocas publicaciones que evalúen la tasa de desnutrición hospitalaria en diversas poblaciones latinoamericanas, usando criterios similares para hacerlo ${ }^{(6,13-15)}$.

El objetivo de este estudio es evaluar la tasa de desnutrición infantil observada al ingreso hospitalario en 9 países latinoamericanos y estimar el impacto de diversas condicionantes que pueden incidir en su desarrollo.

\section{MATERIALES Y MÉTODOS}

Estudio descriptivo transversal, efectuado en 5.366 niños de ambos sexos, ingresados en servicios de salud de Argentina, Chile, Cuba, Guatemala, México, Panamá, Paraguay, Uruguay y Venezuela, durante el período de Julio a Noviembre 2011. Criterios de inclusión: niños entre 1 mes y 18 años de edad, evaluados en las primeras 48 horas de su ingreso, hospitalizados en servicio médico o quirúrgico. La información se obtuvo mediante una encuesta efectuada durante las primeras 48 horas de hospitalización, y en todos los niños se evaluó peso y talla en el momento del ingreso. Se excluyó los recién nacidos, los niños ingresados en unidades de cuidado intensivo, los pacientes politraumatizados $\mathrm{y}$ aquellos niños con registro incompleto de datos antropométricos o con datos inconsistentes.

Para la evaluación nutricional se utilizó el índice de peso para la talla $(\mathrm{P} / \mathrm{T})$ en los niños entre 0 y 2 años, el índice de masa corporal (IMC) para la edad (IMC/E) en los mayores de 2 años, y la talla para la edad (T/E) entre 0 y 18 años. Estos indicadores se evaluaron de acuerdo a los estándares de la Organización Mundial de la Salud (OMS) 2006, para menores de 5 años y OMS 2007 entre 6 y 19 años ${ }^{(13,15)}$.

Se determinó el puntaje Z (DE) de los indicadores $\mathrm{P} / \mathrm{T}$ e IMC para la edad y se consideró, desnutrición cuando el puntaje $\mathrm{Z}$ era inferior a $-2 \mathrm{DE}$, riesgo de desnutrir (desnutrición leve) entre -1,1 y -1,9 DE, eutrofia o normopeso entre-1 DE y $+1 \mathrm{DE}$, sobrepeso entre $+1,1$ y $+1,9$ DE y obesidad cuando era superior a +2 DE. El indicador longitud-talla/edad se utilizó en niños entre 0 y 18 años, considerando su normalidad entre -2 y $+2 \mathrm{DE}$. El diagnóstico de talla baja se hizo cuando la T/E fue inferior a $-2 \mathrm{DE}$, en todas las edades, y el de talla alta cuando este indicador era $\geq+2 \mathrm{DE}^{(5,6,13,16)}$.

Todos los datos se registraron en un formulario único que incluía los siguientes datos: país, edad, sexo, peso, talla, procedencia (urbana o rural), edad y escolaridad de la madre, número de hijos, motivo de ingreso, diagnósticos médicos, peso de nacimiento, pérdida de peso en el último tiempo, síntomas gastrointestinales al momento de la hospitalización y número de hospitalizaciones previas. Los diagnósticos clínicos fueron agrupados según la Clasificación Internacional de Enfermedades (CIE 10) ${ }^{(17)}$. 
Se utilizó el programa WHO Anthro v.3.2.2 para evaluar el estado nutricional de los niños menores de 5 años de edad y el programa WHO Anthro Plus para los niños de 5 a 19 años de edad.

El análisis estadístico se realizó en el programa SPSS versión 24. Se evaluó normalidad de distribución para variables cuantitativas (prueba de KolmogorovSmirnov). Se utilizó la prueba no paramétrica de U de Mann Whitney para comparar variables categóricas de 2 grupos con cualitativas para muestras independientes. Para describir las variables se utilizó la mediana y el rango intercuartil. Para comparar variables categóricas de más de 2 grupos con variables cuantitativas se utilizó la prueba no paramétrica Kruskal -Wallis. Para la asociación de 2 variables categóricas se utilizó la prueba de ChiCuadrado de Pearson.

Se utilizó regresión logística binaria para evaluar el efecto del diagnóstico médico de ingreso, del peso al nacer y del número de hospitalizaciones, procedencia, edad de la madre, escolaridad de la madre y número de hijos sobre el diagnóstico nutricional. También se aplicó regresión logística binaria para evaluar las variables, anteriormente mencionadas, sobre la talla para la edad.

El estudio fue aprobado por el Comité de Ética de cada servicio hospitalario. Se obtuvo el consentimiento informado de los padres o tutores de los pacientes. Los autores declaran no tener conflictos de interés.

\section{RESULTADOS}

Del total de 5.773 niños ingresados, se excluyeron 407 que no registraban peso, talla, fecha de nacimiento, país y/o diagnóstico médico de ingreso, y aquellos cuyos datos fueran inconsistentes. Por lo anterior, en el análisis se incluyeron 5.366 niños.

Las características generales de los niños hospitalizados, incluido el peso al nacer y los antecedentes de edad y natalidad de sus madres se resumen en la Tabla 1. Del total de la muestra un $45,2 \%$ eran mujeres y el $46,5 \%$ eran menores de 2 años. Un $18,3 \%$ del total de pacientes era de procedencia rural y un $81,7 \%$ de procedencia urbana. En relación al estado nutricional, un 50,2\% presentó al ingreso hospitalario un estado nutricional normal, un $15,4 \%$ riesgo de desnutrir o desnutrición leve, un 12,5\% desnutrición, un 14,2\% sobrepeso o riesgo de obesidad, y un 7,7\% obesidad. En cuanto a la talla para la edad, $74,7 \%$ eran de talla normal, $20,5 \%$ de talla baja y $4,9 \%$ de talla alta.

En relación a la escolaridad materna, el 39\% tenían educación básica, 47\% educación media, 12\% estudios superiores o universitarios y sólo un $2 \%$ eran analfabetas.

En la Tabla 2, se presenta la clasificación de las enfermedades que motivaron el ingreso de los niños evaluados. La principal causa de hospitalización fue la patología respiratoria, en el 35,6\% de los casos. Un $9,2 \%$ correspondió a pacientes quirúrgicos.

En la Tabla 3 se observa la distribución de diagnósticos nutricionales por países, destacando una amplia variación en la tasa de desnutrición $(6,3$ a $17,7 \%)$ y en la de riesgo de desnutrir (10,5 a $25,4 \%)$. Cabe señalar también una mayor tasa de malnutrición por exceso (sobrepeso y obesidad) en Chile, México y Panamá.

En la Tabla 4 se observa la distribución de diagnósticos nutricionales según diagnósticos médicos. Destaca la muy alta tasa de desnutrición en enfermedades cardiológicas y gastroenterológicas.

En cuanto a la relación entre la edad y el diagnóstico nutricional, el grupo con mayor prevalencia de desnutrición y riesgo de desnutrir o desnutrición leve, fueron los niños menores de un año (Tabla 5). Por su parte, la mayor prevalencia de talla baja se observó entre los $0-1$ años de edad, con un 25,1\%.

Cuando se relacionó la talla para la edad y la patología de ingreso, las patologías con mayor prevalencia de talla baja fueron las cardiológicas con un $35,4 \%$ ( $p=0,000$ Test de Kruskal-Wallis).

En relación al peso de nacimiento, un 8,5\% de los niños de sexo masculino pesó $4.000 \mathrm{~g}$ o más al nacer, en comparación con las niñas, esto sucedió sólo un $6 \%\left(\mathrm{p}=0,005\right.$ Test de $\left.^{2}\right)$.

No se encontró relación entre la procedencia 
(urbano o rural) con las siguientes variables: diagnóstico nutricional, edad de la madre, edad de la madre al momento del nacimiento, escolaridad de la madre, número de hijos, y peso de nacimiento. Por su parte, a menor escolaridad materna mayor es el número de hijos $\left(\mathrm{p}=0,000\right.$ Test de $\left.{ }^{2}\right)$. Sin embargo, no hubo asociación entre el número de hijos y el diagnóstico de desnutrición ( $p=0,077$ Test de ${ }^{2}$ ).

Las patologías en las que se reportó mayor frecuencia de pérdida de peso previo a la hospitalización fueron las quirúrgicas, oncológicas y gastroenterológicas $(22,3 \%, 22 \%$ y $20,4 \%$ respectivamente), mientras que, en las patologías neurológicas e infecciosas sólo en 1 de cada 6 niños se reportó baja de peso previa al ingreso (Test de Kruskal-Wallis, $\mathrm{p}=0,044$ ).

Se realizó un análisis de regresión logística, asignando como variable dependiente el estado nutricional y como variables independientes el diagnóstico médico, el peso de nacimiento, el número de hospitalizaciones previas, la procedencia urbana o rural, la edad de la madre, su escolaridad y el número de hijos.
Las patologías cardiológicas presentaron un $\mathrm{OR}=$ 1,8 (IC95\% 1,09-2,83) de presentar malnutrición por déficit y en las patologías nefrológicas, neurológicas, respiratorias y traumas o accidentes se observó un OR $=0,5$ (IC95\% 0,35-0,85), 0,4 (IC95\% 0,26-0,61), 0,7 (IC95\% 0,55-0,98) y 0,5 (IC95\% 0,31-0,81), respectivamente. (Figura 1). Los niños con peso de nacimiento entre 600 - $999 \mathrm{~g}$ no presentaron mayor riesgo de desnutrición. Por otra parte, tampoco se observó que a mayor peso de nacimiento tuvieran menor riesgo de presentar alteraciones nutricionales. Sin embargo, los niños con peso de nacimiento entre 600 - $999 \mathrm{~g}$ y entre 1.000 y $1.499 \mathrm{~g}$ presentaron mayor riesgo de presentar malnutrición por exceso (OR = 1,55 IC95\% 1,19-1,67 y OR = 1,6 IC95\% 1,24-1,89, respectivamente). Por otra parte, no se observó que a mayor peso de nacimiento tuvieran mayor riesgo de presentar sobrepeso u obesidad.

Los niños con 4 o más hospitalizaciones previas mostraron mayor riesgo de presentar malnutrición por déficit (OR = 1,7 IC 1,06-2,63). Al considerar la talla para la edad y los diagnósticos médicos de ingreso, se observó que sólo en las patologías cardiológicas existía un mayor riesgo $(\mathrm{OR}=2,3$ IC95\% 1,20-3,25) de presentar talla baja.

Tabla 1. Características generales de niños de 0-18 años de edad, hospitalizados en servicios de salud de 9 países latinoamericanos $(\mathrm{n}=5.366)$

\begin{tabular}{|c|c|c|}
\hline & Mediana $\left(\mathrm{RIC}^{\mathrm{a}}\right)$ & Mínimo-Máximo \\
\hline Edad (años) & $2,41(0,71-8,05)$ & $0,01-18,99$ \\
\hline Z-Score $T / E^{b}$ & $-0,69(-1,72-0,30)$ & $-7,21-5,00$ \\
\hline Z-Score $\mathrm{P} / \mathrm{T}^{\mathrm{c}}-\mathrm{IMC} / \mathrm{E}^{\mathrm{d}}$ & $0,09(-0,97-1,15)$ & $-7,81-7,91$ \\
\hline Edad de la madre (años) & $29(24-36)$ & $13-58$ \\
\hline Edad de la madre al nacimiento (años) & $24,56(20,5-29,7)$ & $9,2-55,1$ \\
\hline Peso al nacer (g) & $3200(2816-3500)$ & $600-5760$ \\
\hline Número de hijos & $2(1-3)$ & $1-19$ \\
\hline $\begin{array}{l}{ }^{\mathrm{a}} \text { Rango intercuartil } \\
{ }^{\mathrm{b}} \text { Talla para la edad } \\
{ }^{\mathrm{c}} \text { Peso para la talla }(<2 \text { años) } \\
{ }^{\mathrm{d}} \text { Índice de masa corporal para la }\end{array}$ & ( 2 años) & \\
\hline
\end{tabular}


Tabla 2. Diagnósticos médicos de ingreso de niños de 0 a 18 años de edad, hospitalizados en 9 países de América Latina.

\begin{tabular}{lcc}
\hline & $\mathbf{n}$ & $\mathbf{\%}$ \\
\hline Respiratorio & 1911 & 35,6 \\
\hline Gastroenterológicos & 761 & 14,2 \\
\hline Infecciosos & 623 & 11,6 \\
\hline Quirúrgicos & 494 & 9,2 \\
\hline Oncológicos y Otros Trastornos Hematológicos & 369 & 6,9 \\
\hline Otros Diagnósticos & 355 & 6,6 \\
\hline Neurológicos & 319 & 5,9 \\
\hline Nefrológicos & 227 & 4,2 \\
\hline Trauma o Accidente & 178 & 3,3 \\
\hline Cardiológicos & 129 & 2,4 \\
\hline Total & $\mathbf{5 3 6 6}$ & $\mathbf{1 0 0}$ \\
\hline
\end{tabular}

Tabla 3. Diagnóstico nutricional de niños de 0 a 18 años de edad al ingreso hospitalario en 9 países de América Latina.

\begin{tabular}{|c|c|c|c|c|c|c|c|c|c|}
\hline & $\begin{array}{c}\text { Argentina } \\
\text { n } \\
(\%)\end{array}$ & $\begin{array}{c}\text { Chile } \\
\text { n } \\
(\%)\end{array}$ & $\begin{array}{c}\text { Cuba } \\
\text { n } \\
(\%)\end{array}$ & $\begin{array}{c}\text { Guatemala } \\
\mathbf{n} \\
(\%) \\
\end{array}$ & $\begin{array}{c}\text { México } \\
\text { n } \\
(\%)\end{array}$ & $\begin{array}{c}\text { Panamá } \\
\text { n } \\
(\%)\end{array}$ & $\begin{array}{c}\text { Paraguay } \\
\text { n } \\
(\%)\end{array}$ & $\begin{array}{c}\text { Uruguay } \\
\qquad \mathbf{n} \\
(\%)\end{array}$ & $\begin{array}{c}\text { Venezuela } \\
\mathbf{n} \\
(\%) \\
\end{array}$ \\
\hline Desnutrición & $\begin{array}{c}35 \\
(13)\end{array}$ & $\begin{array}{c}41 \\
(6,3)\end{array}$ & $\begin{array}{c}226 \\
(12,2)\end{array}$ & $\begin{array}{c}46 \\
(15,9)\end{array}$ & $\begin{array}{c}124 \\
(17,7)\end{array}$ & $\begin{array}{c}107 \\
(13,3)\end{array}$ & $\begin{array}{c}17 \\
(14,9)\end{array}$ & $\begin{array}{c}66 \\
(10,6)\end{array}$ & $\begin{array}{c}7 \\
(11,9)\end{array}$ \\
\hline Riesgo de Desnutrir & $\begin{array}{c}46 \\
(17,1)\end{array}$ & $\begin{array}{c}68 \\
(10,5) \\
\end{array}$ & $\begin{array}{c}290 \\
(15,6)\end{array}$ & $\begin{array}{c}58 \\
(20,1)\end{array}$ & $\begin{array}{c}122 \\
(17,4)\end{array}$ & $\begin{array}{c}121 \\
(15,1)\end{array}$ & $\begin{array}{c}18 \\
(15,8) \\
\end{array}$ & $\begin{array}{c}91 \\
(14,6)\end{array}$ & $\begin{array}{c}15 \\
(25,4)\end{array}$ \\
\hline Normopeso & $\begin{array}{c}147 \\
(54,6)\end{array}$ & $\begin{array}{c}350 \\
(54,1)\end{array}$ & $\begin{array}{c}945 \\
(50,9)\end{array}$ & $\begin{array}{c}146 \\
(50,5)\end{array}$ & $\begin{array}{c}292 \\
(41,6)\end{array}$ & $\begin{array}{c}390 \\
(48,6)\end{array}$ & $\begin{array}{c}56 \\
(49,1)\end{array}$ & $\begin{array}{c}339 \\
(54,2)\end{array}$ & $\begin{array}{c}29 \\
(49,2)\end{array}$ \\
\hline Sobrepeso & $\begin{array}{c}31 \\
(11,5) \\
\end{array}$ & $\begin{array}{c}127 \\
(19,6) \\
\end{array}$ & $\begin{array}{c}244 \\
(13,1)\end{array}$ & $\begin{array}{c}31 \\
(10,7) \\
\end{array}$ & $\begin{array}{c}99 \\
(14,1)\end{array}$ & $\begin{array}{c}117 \\
(14,6) \\
\end{array}$ & $\begin{array}{c}14 \\
(12,3)\end{array}$ & $\begin{array}{c}92 \\
(14,7) \\
\end{array}$ & $\begin{array}{c}5 \\
(8,5)\end{array}$ \\
\hline Obesidad & $\begin{array}{c}10 \\
(3,7)\end{array}$ & $\begin{array}{c}61 \\
(9,4)\end{array}$ & $\begin{array}{l}153 \\
(8,2)\end{array}$ & $\begin{array}{c}8 \\
(2,8)\end{array}$ & $\begin{array}{c}65 \\
(9,3)\end{array}$ & $\begin{array}{c}68 \\
(8,5)\end{array}$ & $\begin{array}{c}9 \\
(7,9)\end{array}$ & $\begin{array}{c}37 \\
(5,9)\end{array}$ & $\begin{array}{c}3 \\
(5,1)\end{array}$ \\
\hline Total & $\begin{array}{c}269 \\
(100)\end{array}$ & $\begin{array}{c}647 \\
(100)\end{array}$ & $\begin{array}{l}1858 \\
(100)\end{array}$ & $\begin{array}{c}289 \\
(100)\end{array}$ & $\begin{array}{c}702 \\
(100)\end{array}$ & $\begin{array}{l}803 \\
100)\end{array}$ & $\begin{array}{c}114 \\
(100)\end{array}$ & $\begin{array}{c}625 \\
(100)\end{array}$ & $\begin{array}{c}59 \\
(100)\end{array}$ \\
\hline
\end{tabular}


Tabla 4. Diagnóstico nutricional por patología de niños de 0 a 18 años de edad al ingreso hospitalario en 9 países de América Latina.

\begin{tabular}{|c|c|c|c|c|c|c|c|c|c|c|c|}
\hline & Respiratoria & $\begin{array}{c}\text { Gastroente- } \\
\text { rológica } \\
\mathbf{n} \\
(\%)\end{array}$ & $\begin{array}{c}\text { Infec- } \\
\text { ciosa } \\
n \\
(\%) \\
\end{array}$ & $\begin{array}{c}\text { Quirúr } \\
\text {-gica } \\
\text { n } \\
(\%)\end{array}$ & $\begin{array}{c}\text { Oncoló- } \\
\text { gica } \\
\mathbf{n} \\
(\%)\end{array}$ & $\begin{array}{c}\text { Otros } \\
\text { Diag. } \\
n \\
(\%) \\
\end{array}$ & $\begin{array}{c}\text { Neuroló- } \\
\text { gica } \\
\mathbf{n} \\
(\%) \\
\end{array}$ & $\begin{array}{c}\text { Nefroló- } \\
\text { gica } \\
\mathbf{n} \\
(\%)\end{array}$ & $\begin{array}{c}\text { Trauma o } \\
\text { Accidente } \\
\mathbf{n} \\
(\%) \\
\end{array}$ & $\begin{array}{l}\text { Cardio- } \\
\text { lógica } \\
\text { n } \\
(\%)\end{array}$ & $\begin{array}{c}\text { Total } \\
\text { n } \\
(\%) \\
\end{array}$ \\
\hline Desnutrición & $\begin{array}{c}224 \\
(11,7) \\
\end{array}$ & $\begin{array}{c}127 \\
(16,7)\end{array}$ & $\begin{array}{c}65 \\
(10,4) \\
\end{array}$ & $\begin{array}{c}53 \\
(10,7) \\
\end{array}$ & $\begin{array}{c}53 \\
(14,4) \\
\end{array}$ & $\begin{array}{c}56 \\
(15,8) \\
\end{array}$ & $\begin{array}{c}22 \\
(6,9) \\
\end{array}$ & $\begin{array}{c}18 \\
(7,9)\end{array}$ & $\begin{array}{c}13 \\
(7,3)\end{array}$ & $\begin{array}{c}38 \\
(29,5) \\
\end{array}$ & $\begin{array}{c}669 \\
(12,5) \\
\end{array}$ \\
\hline $\begin{array}{l}\text { Riesgo de } \\
\text { Desnutrir }\end{array}$ & $\begin{array}{c}326 \\
(17,1)\end{array}$ & $\begin{array}{c}143 \\
(18,8) \\
\end{array}$ & $\begin{array}{c}98 \\
(15,7)\end{array}$ & $\begin{array}{c}63 \\
(12,8) \\
\end{array}$ & $\begin{array}{c}59 \\
(16)\end{array}$ & $\begin{array}{c}47 \\
(13,2) \\
\end{array}$ & $\begin{array}{c}27 \\
(8,5)\end{array}$ & $\begin{array}{c}26 \\
(11,5) \\
\end{array}$ & $\begin{array}{c}22 \\
(12,4)\end{array}$ & $\begin{array}{c}18 \\
(14) \\
\end{array}$ & $\begin{array}{c}829 \\
(15,4)\end{array}$ \\
\hline Normopes & $\begin{array}{l}1028 \\
(53,8) \\
\end{array}$ & $\begin{array}{c}364 \\
(47,8) \\
\end{array}$ & $\begin{array}{c}314 \\
(50,4)\end{array}$ & $\begin{array}{c}229 \\
(46,4)\end{array}$ & $\begin{array}{c}157 \\
(42,5)\end{array}$ & $\begin{array}{c}161 \\
(45,4)\end{array}$ & $\begin{array}{c}181 \\
(56,7)\end{array}$ & $\begin{array}{c}114 \\
(50,2)\end{array}$ & $\begin{array}{c}100 \\
(56,2)\end{array}$ & $\begin{array}{c}46 \\
(35,7) \\
\end{array}$ & $\begin{array}{l}2694 \\
(50,2) \\
\end{array}$ \\
\hline Sobrepeso & $\begin{array}{c}217 \\
(11,4)\end{array}$ & $\begin{array}{c}83 \\
(10,9)\end{array}$ & $\begin{array}{c}91 \\
(14,6)\end{array}$ & $\begin{array}{c}91 \\
(18,4)\end{array}$ & $\begin{array}{c}63 \\
(17,1) \\
\end{array}$ & $\begin{array}{c}68 \\
(19,2)\end{array}$ & $\begin{array}{c}60 \\
(18,8)\end{array}$ & $\begin{array}{c}46 \\
(20,3)\end{array}$ & $\begin{array}{c}24 \\
(13,5) \\
\end{array}$ & $\begin{array}{c}17 \\
(13,2)\end{array}$ & $\begin{array}{c}760 \\
(14,2)\end{array}$ \\
\hline Obesidad & $\begin{array}{c}116 \\
(6)\end{array}$ & $\begin{array}{c}44 \\
(5,8)\end{array}$ & $\begin{array}{c}55 \\
(8,8)\end{array}$ & $\begin{array}{c}58 \\
(11,7) \\
\end{array}$ & $\begin{array}{c}37 \\
(10)\end{array}$ & $\begin{array}{c}23 \\
(6,5)\end{array}$ & $\begin{array}{c}29 \\
(9,1)\end{array}$ & $\begin{array}{c}23 \\
(10,1)\end{array}$ & $\begin{array}{c}19 \\
(10,7)\end{array}$ & $\begin{array}{c}10 \\
(7,8)\end{array}$ & $\begin{array}{l}414 \\
(7,7)\end{array}$ \\
\hline Total & $\begin{array}{l}1911 \\
(100)\end{array}$ & $\begin{array}{c}761 \\
(100)\end{array}$ & $\begin{array}{c}623 \\
(100)\end{array}$ & $\begin{array}{c}494 \\
(100)\end{array}$ & $\begin{array}{c}369 \\
(100)\end{array}$ & $\begin{array}{c}355 \\
(100)\end{array}$ & $\begin{array}{c}319 \\
(100)\end{array}$ & $\begin{array}{c}227 \\
(100)\end{array}$ & $\begin{array}{c}178 \\
(100)\end{array}$ & $\begin{array}{c}129 \\
(100)\end{array}$ & $\begin{array}{l}5366 \\
(100)\end{array}$ \\
\hline
\end{tabular}

Tabla 5. Diagnóstico nutricional por rango etario de niños de 0 a 18 años de edad al ingreso hospitalario en 9 países de América Latina.

\begin{tabular}{lcccc|c}
\hline & $\mathbf{0 - 1}$ años & $\mathbf{2 - 5}$ años & $\mathbf{6 - 9}$ años & $\mathbf{1 0 - 1 8}$ años & Total \\
\hline Desnutrición & $\mathbf{n}(\mathbf{\%})$ & $\mathbf{n ~ ( \% )}$ & $\mathbf{n ~ ( \% )}$ & $\mathbf{n ~ ( \% )}$ & $\mathbf{n}(\mathbf{\%})$ \\
\hline Riesgo de Desnutrir & $391(15,7)$ & $78(6,9)$ & $89(12,8)$ & $111(10,5)$ & $669(12,5)$ \\
\hline Normopeso & $448(18,0)$ & $162(14,4)$ & $92(13,2)$ & $127(12,1)$ & $829(15,4)$ \\
\hline Sobrepeso & $1315(52,7)$ & $546(48,6)$ & $308(44,3)$ & $525(49,9)$ & $2694(50,2)$ \\
\hline Obesidad & $248(9,9)$ & $203(18,1)$ & $112(16,1)$ & $197(18,7)$ & $760(14,2)$ \\
\hline Total & $93(3,7)$ & $134(11,9)$ & $94(13,5)$ & $93(8,8)$ & $414(7,7)$ \\
\hline
\end{tabular}

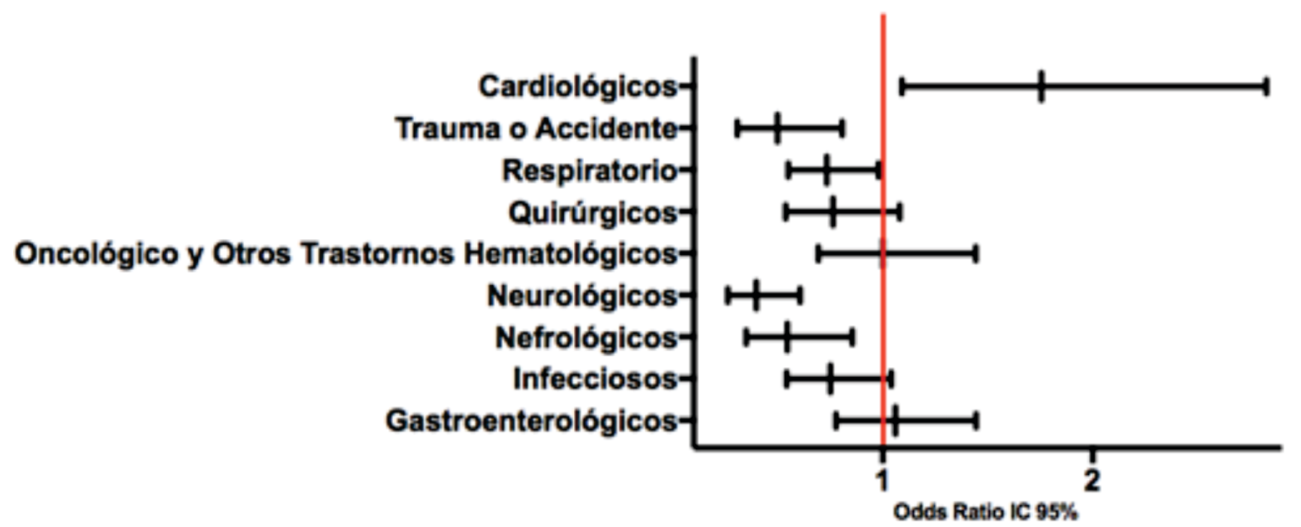

Figura 1. Razón de Chances (OR IC 95\%) para diagnósticos médicos de ingreso en pacientes normopeso versus desnutridos. 


\section{DISCUSIÓN}

Este trabajo muestra la prevalencia de desnutrición al ingreso hospitalario en 32 servicios de salud de 9 países de Latinoamérica y los principales factores que contribuyen a la desnutrición de pacientes pediátricos hospitalizados en estos centros. Un estudio publicado en 2003, evaluó la desnutrición en pacientes hospitalizados en 13 países latinoamericanos. Sin embargo, éste fue efectuado en adultos y con metodología diferente a nuestro estudio ${ }^{(18)}$.

$\mathrm{Al}$ igual que en otras poblaciones, en este estudio el mayor porcentaje de niños desnutridos o con riesgo de desnutrir se presenta en el grupo de lactantes menores de un año. Este grupo es el más propenso a presentar grandes trastornos en su desarrollo producto de la desnutrición ${ }^{(19,20)}$. Las patologías cardiacas presentaron un alto riesgo de desnutrición, siendo las patologías con mayores tasas de malnutrición por déficit. Por otra parte, los niños con cardiopatías tienen 2,3 veces más probabilidades de presentar talla baja, al igual como se ha descrito en la literatura. Estas enfermedades cursan con hipoxemia, inadecuada ingesta calórica, aumento del gasto energético, malabsorción intestinal, déficit de nutrientes específicos, acidosis metabólica, todos los cuales contribuyen a la desnutrición y detención de crecimiento ${ }^{(21,22)}$.

Un 4,9\% de la población en estudio presentó talla alta, situación que se observó con mayor frecuencia en los ingresos con enfermedad neurológica $(7,4 \%)$. En estos niños hay dificultades para realizar mediciones de talla, y muchas veces ésta se estima con mediciones por segmentos, pudiendo ser un parámetro poco confiable en este grupo.

Según los datos recolectados, en este estudio más niños que niñas fueron macrosómicos (peso $>4000$ g), un hecho que ha sido descrito previamente. Acorde la literatura, existe una relación entre el peso de nacimiento y la edad de la madre, ya que a mayor edad materna mayor peso de nacimiento, sin embargo, esto no incidió en el estado nutricional al momento del ingreso hospitalario.

A mayor educación materna menor es la cantidad de hijos. Del total de la muestra ninguna madre con estudios superiores o universitarios tuvo más de 7 hijos y el $95,4 \%$ tuvo tres hijos o menos. Por otra parte, las madres analfabetas tienen la mayor cantidad de hijos. Las madres con educación deficiente tienden a presentar mayor cantidad de hijos, y un buen nivel de educación mejora los conocimientos sobre nutrición y el estado de salud en general, en la medida que se les otorga a las personas conocimientos, capacidades y prácticas para guiar y controlar conductas y hábitos alimentarios adecuados ${ }^{(1,2,19)}$. Sin embargo, como se mencionó anteriormente, a pesar que la educación materna es un factor bien estudiado y descrito en la influencia sobre el estado nutricional del niño, como muestra el Panorama de la Seguridad Alimentaria y Nutricional de América Latina y el Caribe, en el presente estudio eso no se evidenció de manera significativa. Esto podría deberse al bajo porcentaje de madres analfabetas que participaron del estudio. Acorde a la literatura, un niño que vive en una zona rural tiene más riesgo de sufrir desnutrición que un niño que vive en zona urbana y más probabilidades de sufrirla si es de origen indígena. En el presente estudio no se tenían datos del origen étnico de los menores, pero en relación a la procedencia no se obtuvo diferencias significativas, lo que puede deberse al bajo porcentaje de individuos de origen rural versus urbano de la muestra $(18,3 \%$ vs $81,7 \%)$. Estos porcentajes no serían representativos de la región en estudio, ya que por ejemplo en Guatemala el porcentaje de pacientes que viven en zonas urbanas es de un $42 \%$ y en zonas rurales de un $58 \%{ }^{(1-3,9,23)}$.

Según varias publicaciones, el diagnóstico médico y la pérdida de peso se relacionan significativamente. Las patologías con mayor reporte de pérdida de peso fueron las quirúrgicas, gastroenterológicas y oncológicas. La pérdida de peso en pacientes con cáncer es frecuente, observándose que un 30 a 80\% de los pacientes con cáncer, baja de peso. La prevalencia de desnutrición en este mismo grupo también es elevada, oscilando entre 40 a $80 \%$, siendo mayor en pacientes hospitalizados, en el presente estudio se observó que sólo un 30,4\% de los niños con estas patologías presentaba malnutrición por déficit.

Si bien las únicas patologías con un factor de riesgo de presentar desnutrición o riesgo de desnutrir son 
las cardiopatías y los trastornos hematológicos, otras enfermedades como las nefrológicas, neurológicas, respiratorias presentaron OR menores a 1, un factor supuestamente protector ante posibles deficiencias nutricionales. Esto parece contradictorio y se podría explicar por diferentes causas. Algunas enfermedades cursan con edema, el que no siempre es bien registrado, lo que podría dificultar la interpretación de la medición del peso. Además un elevado porcentaje de los pacientes que ingresaron por patologías denominadas como nefrológicas correspondían a infecciones del tracto urinario bajo, la cual tiene baja probabilidad de presentar malnutrición por déficit. Como se mencionó anteriormente, en las patologías neurológicas hay una mayor dificultad para tomar medidas antropométricas, por lo que las cifras y valores pueden ser poco confiables. Por otra parte, la mayoría de las enfermedades respiratorias eran agudas y además era la primera hospitalización de cada niño, por lo tanto, la probabilidad de presentar malnutrición por déficit a causa de su enfermedad sería menor.

El peso de nacimiento es un factor determinante para presentar malnutrición por exceso. Es interesante analizar lo sucedido al realizar una regresión logística, en la que se observó que los niños con el antecedente de haber tenido muy bajo peso de nacimiento, tienen alto riesgo de presentar obesidad a medida que crecen, tal como ha sido descrito por Barker respecto a la programación fetal ${ }^{(4,24,25)}$.

Como se ha estimado, la cantidad de hospitalizaciones previas incide en el estado nutricional del niño al momento de la hospitalización. En el presente estudio se evidencia que a mayor cantidad de hospitalizaciones aumenta en 1,7 veces la probabilidad de observar desnutrición y riesgo de desnutrir $^{(26,27)}$.

Cabe señalar una alta tasa de malnutrición por sobrepeso y exceso al ingreso hospitalario en algunos servicios de salud, sobre todo en Chile, México y Panamá, lo cual es un reflejo de la situación observada en la región en la última década. Por lo tanto, es importante identificar a todos los niños con malnutrición al ingreso hospitalario, sea por déficit o por exceso, para brindar una efectiva intervención nutricional, estar atento a las prácticas que pueden contribuir a agravar una malnutrición preexistente y efectuar un seguimiento nutricional al alta hospitalaria $^{(28,29)}$.

Este es el primer estudio que muestra la prevalencia de desnutrición pediátrica al ingreso hospitalario, en varios centros clínicos de Latinoamérica, y los factores asociados a ésta. Destaca la variabilidad del estado nutricional al comparar los distintos países. La prevalencia de desnutrición infantil al ingreso hospitalario fue del $12,5 \%$, relacionada especialmente a patología cardíaca y a múltiples hospitalizaciones previas.

A pesar del gran número de niños reclutados, no se debe inferir que este estudio sea representativo de la realidad de la Región, pues sólo incluye a 9 países latinoamericanos. Se requiere de más estudios, con poblaciones representativas y en lo posible con seguimiento de la evolución intrahospitalaria, para tener una certera visión de la realidad de la desnutrición pediátrica en los hospitales de Latinoamérica.

\section{CONCLUSIONES}

La prevalencia de desnutrición en niños al ingreso hospitalario es moderada y se relaciona especialmente a patología cardiológica y al antecedente de varias hospitalizaciones previas.

\section{CONTRIBUCIÓN DE AUTORÍA}

M. Isabel Hodgson: Redacción del manuscrito, Recolección de los datos, Análisis de datos y resultados, Revisión y aprobación de la versión final, Coordinación local en el país correspondiente, Asumir la corresponsabilidad de todos los aspectos del manuscrito.

Raquel Maciques: Concepción y diseño del estudio, Recolección de los datos, Coordinación local en el país correspondiente.

Adriana Fernández: Recolección de los datos, Revisión y aprobación de la versión final, Coordinación local en el país correspondiente. 
Ana Inverso: Recolección de los datos, Revisión y aprobación de la versión final, Coordinación local en el país correspondiente.

Marta P. Márquez: Recolección de los datos, Coordinación local en el país correspondiente.

Francisco Lagrutta: Recolección de los datos, Coordinación local en el país correspondiente.

Marta Sanabria: Recolección de los datos, Revisión y aprobación de la versión final, Coordinación local en el país correspondiente.

Liliana Vera: Recolección de los datos, Coordinación local en el país correspondiente.

Rina Peña: Recolección de los datos, Coordinación local en el país correspondiente

Lázaro Alfonso: Recolección de los datos, Revisión y aprobación de la versión final, Coordinación local en el país correspondiente.

\section{REFERENCIAS}

1. ONU/OPS/OMS. América Latina y el Caribe. Panorama de la seguridad alimentaria y nutricional. 2016.

2. Jiménez-Benítez D, Rodríguez-Martín A, JiménezRodríguez R. Análisis de determinantes sociales de la desnutrición en Latinoamérica. Nutr Hosp. 2010; 25 (supl 3):18-25.

3. Guardiola J, González-Gómez F. La influencia de la desigualdad en la desnutrición de América Latina; una perspectiva desde la economía. Nutr Hosp. 2010; 25 (supl 3):38-43.

4. Barker DJ. Barker DJ. The origins of the developmental origins theory. J Intern Med. 2007; 261(5): 412-7. doi: https://doi.org/10.1111/j.1365-2796.2007.01809.x

5. Mehta NM, Corkins MR, Lyman B, Malone A, Goday PS, Carney LN, et al. Defining Pediatric Malnutrition: A Paradigm Shift Toward Etiology-Related Definitions. J Parenter Enter Nutr. 2013; 37(4):460-81. doi: https://doi.org/10.1177/0148607113479972
Rubens Feferbaum: Concepción y diseño del estudio.

Camila Kluever: Redacción del manuscrito, Análisis de datos y resultados.

\section{AGRADECIMIENTOS}

Argentina: Alberti MJ, Fabeiro M, D`Biassi P, Fain H, Martínez S. Chile: Barja S, Araya F, Cordero ML, Niklitschek J, Pool C, Baeza C, Bustos E, Dinamarca K, Tinoco AC, Velandia S. Cuba: Jiménez García R, Senra Reyes LM, Rodríguez Hernández E, Cordero Herrera M, García Peña OL, Caballero Pineda E, Pérez González Y, Sánchez Cabrera YJ. Guatemala: Pimentel V. México: Avilés Vlaverde A, Baldwin Monroy D, Gómez Anel. Panamá: Bejarano R, Alvarado K, Cención J, Araúz V, Castillo F, Ríos K, de la Cruz A. Paraguay: Medina C. Uruguay: Tanzi MN. Venezuela: Soto I, López A.

6. Becker P, Carney LN, Corkins MR, Monczka J, Smith E, Smith SE, et al. Consensus Statement of the Academy of Nutrition and Dietetics/American Society for Parenteral and Enteral Nutrition: Indicators recommended for the identification and documentation of pediatric malnutrition (undernutrition). Nutr Clin Pract. 2015; 30(1):147-61. doi: https://doi.org/10.1177/0884533614557642

7. Joosten KF, Hulst JM. Nutritional screening tools for hospitalized children : Methodological considerations. Clin Nutr. 2014; 33(1): 1-5. https://doi.org/10.1016/j.cln u.2013.08.002

8. Sarni RO, Carvalho MF, do Monte CM, Albuquerque ZP, Souza FI. Anthropometric evaluation, risk factors for malnutrition, and nutritional therapy for children in teaching hospitals in Brazil. J Pediatr (Rio J). 2009; 85(3):223-228. doi: https://doi.org/10.2223/jped.1890

9. Joosten KF, Hulst JM. Malnutrition in pediatric hospital patients: current issues. Nutrition. 2011; 27(2):133-7. doi: https://doi.org/10.1016/j.nut.2010.06.001 
10. Kac G, García Alvear JL. Epidemiología de la desnutrición en Latinoamérica: situación actual. Nutr Hosp. 2010; 25(Supl.3):50-6.

11. Macías-Rosales R, Vásquez EM, Larrosa A, Rojo M, Bernal A, Romo H. Secondary malnutrition and overweight in a pediatric referral hospital: Associated factors. Pediatr Gastroenterol Nutr. 2009; 48(2):226-32. doi: https://doi.org/10.1097/mpg.0b013e31818de182

12. Pawellek I, Dokoupil K, Koletzko B. Prevalence of malnutrition in paediatric hospital patients. Clin. Nutr 2008; 27:72-76. doi: https://doi.org/10.1016/j.clnu.2007.11.001

13. World Health Organization. WHO. WHO Child Growth Standards: Length/height-for-age, weight-for-age, weight-for-length, weight-for-height and body mass indexfor-age: Methods and development. WHO, Geneva; 2007.

14. Gomila AA, De Grandis ES, Visconti GB, Montero SB, Bertero MI, Marietti LG. Estado nutricional en niños internados en salas de cuidado mínimo. Hospital de Niños de la Santísima Trinidad. Córdoba. Arch Argent Pediatr. 2009; 107(1): 37-42. doi: https://doi.org/10.1590/s032500752009000100009

15. Beer SS, Juarez MD, Vega MW, Canada NL. Pediatric Malnutrition: Putting the New Definition and Standards Into Practice. Nutr Clin Pract. 2015; 30(5):609-24. doi: https://doi.org/10.1177/0884533615600423

16. Ministerio de Salud de Chile. Patrones de crecimiento para la evaluación nutricional de niños, niñas y adolescentes desde el nacimiento hasta los 19 años de edad. Ministerio de Salud: Departamento de Nutrición y Alimentos; 2017.

17. Ramos Martín-Vegue AJ, Vázquez-Barquero JL, Herrera Castanedo S. CIE-10 (I): Introducción, historia y estructura general. Papeles Médicos. 2002; 11(1):24-35.

18. Correia MITD, Campos ACL. ELAN Cooperative Study. Prevalence of hospital malnutrition in Latin America : The Multicenter ELAN Study. Nutrition 2003; 19(10):823-5. doi: https://doi.org/10.1016/s0899-9007(03)00168-0

19. Ortiz-Andrellucchi A, Peña Quintana L, Albino Beñacar A, Monckeberg Barros F, Serra-Majem L. Desnutrición infantil, salud y pobreza: Intervención desde un programa integral. Nutr Hosp. 2006; 21(4): 533-41.
20. Abdelhadi RA, Bouma S, Bairdain S, Wolff J, Legro A, Plogsted S, et al. Characteristics of Hospitalized Children With a Diagnosis of Malnutrition: United States, 2010. J Parenter Enter Nutr. 2016; 40(5):623-35. doi: https://doi.org/10.1177/0148607116633800

21. García Algas F, Rosell Camps A. Nutrición en el lactante con cardiopatía congénita. p.153-162. En: Protocolos Diagnósticos y Terapéuticos en Cardiología Pediátrica. Sociedad Española de Cardiología pediátrica;2015.

22. Oliva Jaramillo EF, Sandoval Pinzon LA, Madrid AJ. Antropometría en niños con cardiopatías congénitas del Hospital Universitario del Valle “Evaristo García” de Cali, Colombia. Rev Gastrohnup. 2013; 15(1):4-9.

23. Martínez R, Fernández A. Desnutrición infantil en América Latina y el Caribe. CEPAL, UNICEF. Boletín Desafíos. 2006;(2):12.

24. Ernst KD, Radmacher PG, Rafail ST, Adamkin DH. Postnatal malnutrition of extremely low birth-weight infants with catch-up growth postdischarge. J Perinatol. 2003; 23:477-82. doi: https://doi.org/10.1038/sj.jp.7210974

25. Barrera Reyes R, Fernandez Carrocera LA. Programación metabólica fetal. Perinatol Reprod Hum. 2015; 29(3):99-105.

26. Hecht C, Weber M, Grote V, Daskalou E, Dell'Era L, Flynn D, et al. Disease associated malnutrition correlates with length of hospital stay in children. Clin Nutr. 2015; 34(1):53-9. doi: https://doi.org/10.1016/j.clnu.2014.01.003

27. Hartman C, Shamir R, Hecht C, Koletzko B. Malnutrition screening tools for hospitalized children. Curr Opin Clin Nutr Metab Care. 2012; 15(3):303-9. doi: https://doi.org/10.1097/mco.0b013e328352dcd4

28. Velandia S. Hodgson MI, Le Roy C. Evaluación nutricional en niños hospitalizados en un Servicio de Pediatría. Rev. Chil. Pediatr. 2016; 87(5):359-365. soi: https://doi.org/10.1016/j.rchipe.2016.05.001

29. McCarthy A, Delvin E, Marcil V, Belanger V, Marchand $\mathrm{V}$, Boctor D, et al. Prevalence of Malnutrition in Pediatric Hospitals in Developed and In-Transition Countries: The Impact of Hospital Practices. Nutrients. 2019; 11(2):236. doi: https://doi.org/10.3390/nu11020236 